decisively surpassed by mapping 795-nm photons generated from a rubidium atomic ensemble into telecom-band 1,367-nm photons using a coherent four-wave mixing process $^{7}$. Optical-fibre losses are about $0.3 \mathrm{~dB} \mathrm{~km}^{-1}$ at $1,367 \mathrm{~nm}$, but are close to 10 times as high near the $800-\mathrm{nm}$ wavelength used previously. The change in wavelength thereby extends the propagation distance by close to a factor of 10 with the same optical losses.

The second exciting development in the work of Radnaev et al. is the demonstration of an increase in memory time of the atomic ensemble from a previous best of less than $10 \mathrm{~ms}$ to more than $100 \mathrm{~ms}$. This order-of-magnitude improvement is due to an ingenious method for compensating light-induced Stark shifts in the optical lattice holding the atomic ensemble. A far-detuned light beam provides a trapping potential that scales inversely with the difference in frequency between the trapping laser and the atomic resonance. At the same time the rate at which atoms scatter photons, which leads to heating and decoherence of the stored quantum information, scales inversely with the square of the frequency difference. Traps that operate at large frequency differences therefore suffer very little decoherence from photon scattering. There is, however, a caveat. The trapping light provides a slightly different potential for the two states the quantum information is stored in, and the potential difference changes ever so slightly as atoms move about in the trap. This leads to motional decoherence of the atoms, which has limited coherence times to under $10 \mathrm{~ms}$ in previous experiments with trapped atomic ensembles.

Radnaev et al. have introduced a new approach for compensating the differential potential by adding an extra laser beam that combines with the trapping laser to produce a twophoton resonance to an excited state. The compensation is not perfect but has led to a 10 -fold increase in coherence time, with even better performance projected for future experiments with higher laser power.

These advances are likely to become widely used in future demonstrations of long-distance quantum-state transfer. Although telecom-band photons are optimal for transmission through optical fibres, shorter-wavelength visible photons are preferred for free-space transmission, to satellites for example. Thus the demonstrated ability to transfer quantum states between photons of different wavelengths is likely to be valuable for producing heterogeneous quantum links. Although a "quantum internet" is still far from being built, the advances of Radnaev et al. add new capabilities that will be important components of the quantum infrastructure of the future.

Mark Saffman is in the Department of Physics, University of Wisconsin-Madison, 1150 University Avenue, Madison, Wisconsin 53706, USA.

e-mail:msaffman@wisc.edu

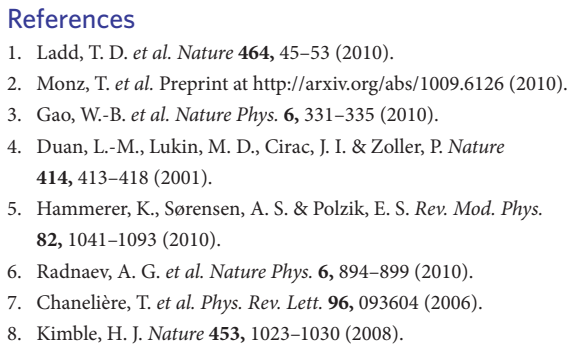

\title{
METROLOGY
}

\section{Just a matter of time}

Before the spookiness of quantum mechanics, came the queerness of relativity. We learned that, contrary to everyday experience, time and space are dependent on our frame of reference. $A$ consequence is that two clocks positioned at different points in a gravitational field, or moving at different speeds, tick at different frequencies. This time dilation is now discernable between two clocks separated by only one metre in height or moving at a speed of less than $10 \mathrm{~ms}^{-1}$ - if, that is, you have access to world's most accurate time pieces (C.-W. Chou et al. Science 329, 1630-1633; 2010).

Both special and general relativity give rise to a form of time dilation. In special relativity, it is a consequence of the constant relative speed of two observers; each sees time progress more slowly for the other. In general relativity, gravity is the underlying cause. The clock at a higher point in the field runs faster than a lower one.

These effects are both tiny. Take the example of the 1971 experiment by Joseph Hafele and Richard Keating. They

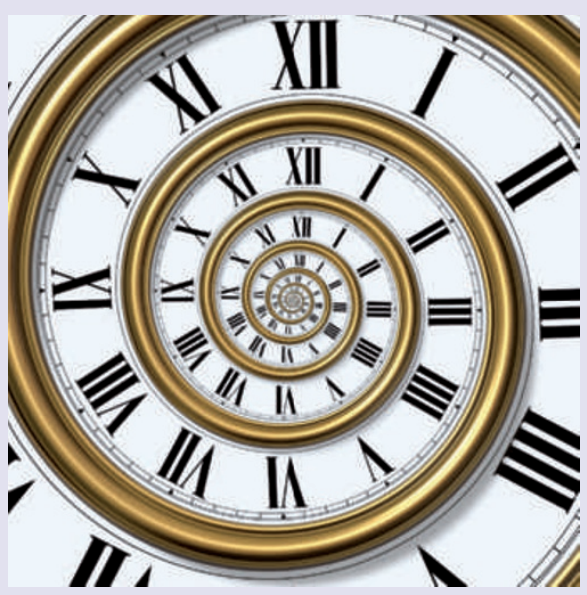

placed clocks on commercial airliners that were circumnavigating the globe. A clock flying westwards at a few hundred metres per second and at an altitude of approximately $10 \mathrm{~km}$ was just $273 \mathrm{~ns}$ ahead of a reference clock on the ground after 50 hours of flying time. They calculated that about $65 \%$ of this was an effect of gravity and the remainder due to the relative motion.

Atomic clocks are needed to measure such small changes. These keep time using stable atomic transitions. Whereas Hafele and Keating used a microwave-frequency transition in caesium atoms, Chin-Wen Chou and colleagues use state-of-the-art opticalfrequency atomic clocks, which keep time at least ten times more accurately.

They compared two aluminium-ionbased clocks connected by an optical fibre. As one was raised by $33 \mathrm{~cm}$ with respect to the other, the frequency of the ticks changed by one part in $2.5 \times 10^{16}$, a change that the optical clocks were just sensitive enough to measure. To measure the effect of motion, rather than move the whole clock, the team applied an electric field, making the aluminium ions vibrate. Again, the apparatus was sensitive enough to measure a time dilation, even when the atoms were moving at just $5 \mathrm{~ms}^{-1}-$ slower than a running athlete.

DAVID GEVAUX 